\title{
Strong Calcite-Like Spectra Cathodoluminescence Emission from Allende Meteorite Cai Phases
}

\author{
Javier Garcia-Guinea', \\ Laura Tormo', \\ Oscar Azumendi², \\ Javier Ruiz ${ }^{2}$, \\ and Virgilio Correcher ${ }^{3}$ \\ ${ }^{1}$ Museo Nacional Ciencias \\ Naturales, CSIC, Madrid, Spain \\ ${ }^{2}$ Dept. Geodinamica, Fac. C. \\ Geologicas, Univ. Complutense, \\ Madrid, Spain \\ ${ }^{3}$ CIEMAT, Madrid, Spain
}

\begin{abstract}
Calcium-aluminum-rich inclusions (CAIs) of Allende CV3 chondrite were studied by Environmental Scanning Electron Microscopy (ESEM), Energy Dispersive Spectrometry (EDS), Backscattering (BS), and Spectra Cathodoluminescence (CL). CAI minerals show spectra CL curves exceeding the 450,000 a.u. with a large homogeneity along the white inclusions. CL curve features fit perfectly with terrestrial patterns of stressed specimens of weathered marble and limestone in which hydroxyl groups may be involved. Crypto-clusters of $\mathrm{CaCO}_{3}$, undetectable by means of EPMA, EDS, and Micro-Raman techniques, coupled to the CAI phases are inferred from the spectral CL data and from the carbon and calcium contents provided by the EDS probe.
\end{abstract}

KEYWORDS Allende, Ca-Al inclusions (CAls), calcite, cathodoluminescence, meteorite, spectra

\section{INTRODUCTION}

Allende CV3 chondrite fragments have been extensively studied during the past 40 years, including by thermoluminescence (TL) and cathodoluminescence (CL) techniques, being remarkable in obtaining 780 hits introducing the topic $=$ Allende and topic $=$ Meteorite in the databases $=$ SCI-expanded of the ISE Web of Knowledge. This fact could be explained by the meteorite complexity from the perspectives of geology, astronomy and materials sciences, together with the advances of the analytical facilities allowing restudy specimens with improved techniques. Calcium-aluminum-rich inclusions (CAIs) found in the Allende CV3 chondrite are the oldest known materials formed in the solar system. ${ }^{[1]}$ CAIs are composed of a suite of Caand Al-rich minerals such as spinel, melilite, pyroxene, and anorthite, which are predicted to be the first to condense from a cooling gas of solar composition. ${ }^{[2]}$ Powdered fragments of the Allende meteorite, which fell in 1969 on Mexico, were initially studied from a thermoluminescent point of view (TL) to delineate its passage through the atmosphere. ${ }^{[3]}$ A large number of Allende studies have focused on their Ca-Al-rich inclusions. ${ }^{[4-6]}$ The strong luminescence sensitivity of these materials continues being the subject of 
TL studies on pulverized aliquots, that is, luminescence techniques nonspectrally and nonspatially resolved ${ }^{[7]}$ In the 1990 s, improved CL imaging devices coupled to spectra CL detectors allowed performing more precise measurements on Allende chondrite minerals. ${ }^{[8]}$ Recent studies on CL and Electron Probe Micro-Analysis (EPMA) of anorthite in Allende CAIS differentiate CL-bright and CL-dark domains, being those dark areas enriched in $\mathrm{MgO}(0.19$ vs. $0.11 \mathrm{wt} \%)$ and $\mathrm{Na}_{2} \mathrm{O}(0.12$ vs. $0.08 \mathrm{wt} \%)$ compared to CL-bright domains, to discriminate differences in alteration histories. ${ }^{[9]}$ Minerals identified in Allende CAIS are anorthite, diopside, fassaite, hibonite, melilite, spinel, perovskite, grossular, monticellite nepheline, sodalite, and wollastonite ${ }^{[10]}$ Occasionally, calcite is also cited in Allende CAI as a secondary phase together with hydrous silicate glass. ${ }^{[11]}$ Concerning the spectra luminescence of calcite, we previously studied the crystal field effects on the luminescence of manganese in carbonate lattices, focusing the work on the orange-red spectrum region. ${ }^{[12]}$ Nowadays the publications on calcite luminescence continue giving emphasis to the importance of the $\mathrm{Mn}^{2+}$ activators in $\mathrm{Ca}^{2+}$ positions ${ }^{[13]}$ since many calcite specimens exhibit large red-orange luminescence emission and negligible or no luminescence in the UV-blue region of the spectrum. However, it is not the case of stressed calcite samples with hydroxyl groups, for example, limestone with maxima at $380 \mathrm{~nm}^{[14]}$ or weathered marble. ${ }^{[15]}$ Similar luminescent behavior is observed in stressed calcite chips under cryogenic conditions during the experimental CL measurements in which calcite spectra show enlargement of the CL emission in the UV-blue region of the spectrum. The present work is important since it includes strong spectra CL of calcite, not previously studied in Allende meteorite, together with terrestrial counterparts. These highsensitivity spectra CL of calcite will be useful for both further researching paths, nondestructive CL analyses of meteorites, and studies on relationships between calcite defects and luminescence emission in the ubiquitous terrestrial materials calcite, marble, and limestone.

\section{OBJECTIVE}

Objective of this work is to study the spectra luminescence emission of the Allende $\mathrm{Ca}-\mathrm{Al}$-rich inclusions under the ESEM-EDS-CL system. The recorded spectra CL of these mineral phases reach photonic emissions up to half of a million arbitrary units, exhibiting a great homogeneity by chemically different calcium-aluminum-silicate phases as observed under both EDS probe and backscattering image.

\section{MATERIALS AND METHODS}

These works must be performed by nondestructive methods on an Allende CV3 Chondrite from the Museo Nacional Ciencias Naturales (MNCN) of Madrid. The microscopically observation, chemical analyses, and CL measurements, such as spectral curves, panchromatic plots, and monochromatic plots were performed by ESEM-EDS using an ESEM Inspect FEI Quanta microscope (FEI, Netherlands) on the Allende Meteorite, at low vacuum mode without coating to keep the way open out to the CL emission, using a CL detector Gatan MonoCL3 series 900 (Gatan, UK) with a PA-3 photomultiplier (PMT) attached to the ESEM. The PMT covers a spectral range from $185 \mathrm{~nm}$ to $850 \mathrm{~nm}$, being most sensitive in the blue parts of the spectrum. A retractable parabolic diamond mirror and a photomultiplier tube are used to collect and amplify luminescence. Samples were positioned $\sim 15 \mathrm{~mm}$ beneath the bottom of the CL mirror assembly. The excitation for CL measurements was provided at $25 \mathrm{kV}$ electron beam. The high sensitivity of the photomultiplier tube gives much improved noise performance over the standard tubes. The very high quantum efficiency of the PMT together with the high luminescence sensitivity of the phases of Allende CAIS produces undesirable signal saturations since the specimen emits light above 450,000 a.u. We must recalculate the analytical conditions for the CL measurements to circumvent the PMT saturation by reducing the spot diameter of the electron gun, controlling the resultant spectra CL curve (Fig. 1). Initially, the maxima CL emission at approximately $340 \mathrm{~nm}$ recorded on silicate samples together with the intrinsic crystalline strain of the CAI minerals as produced by nonbridging-oxygen or silicon vacancy hole centers associated with $\mathrm{Si}-\mathrm{O}$ strained structures misled us, as we observed in many other silicate cases under the cryogenic stress of the analytical measurements. ${ }^{[16]}$

\section{RESULTS}

Figure 2 shows a repolished section of the Allende CV3 chondrite fragment of the MNCN institution 

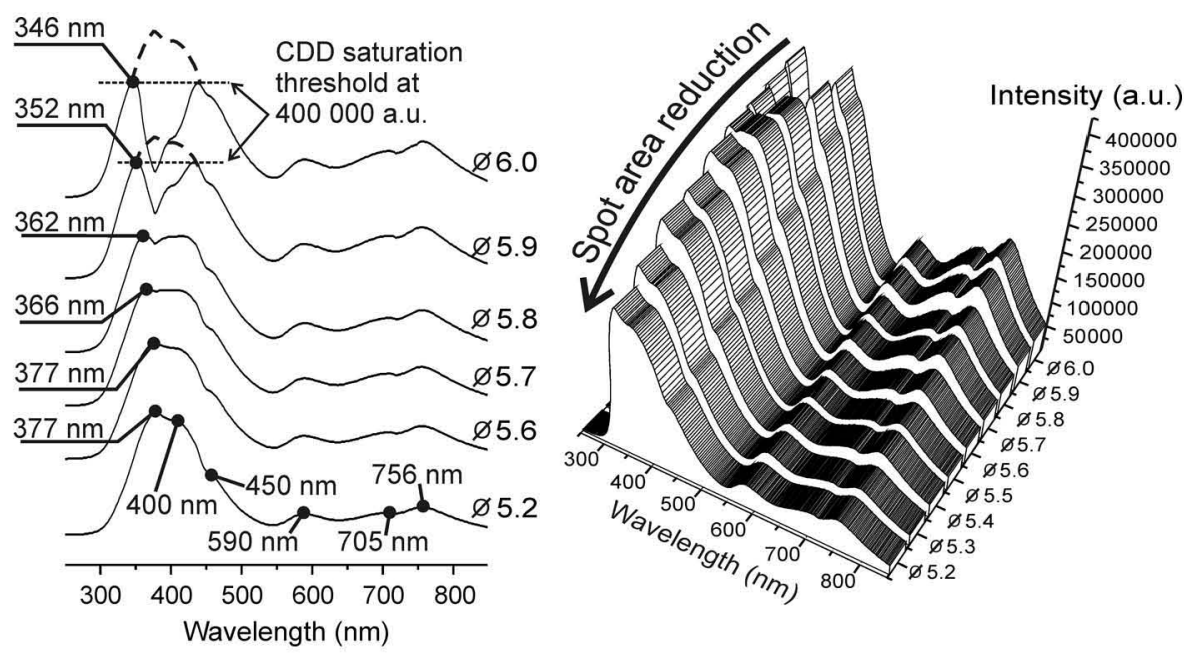

FIGURE 1 Spectra CL curves of an Allende CAI phase taken at different diameter spots of electron gun. Note the decrease of the CL emission as a function of the spot reduction. The visible spectral shift of the maxima peak changes from 346 to $377 \mathrm{~nm}$.

(Madrid, Spain) together with the analytical routine performed under ESEM-EDS.

In accordance with simplest CL theory assumptions to obtain exciting light emission from intrinsic relaxations, impurities, and imperfections in the outer few microns of the surface of insulating and semiconducting materials, we selected the white

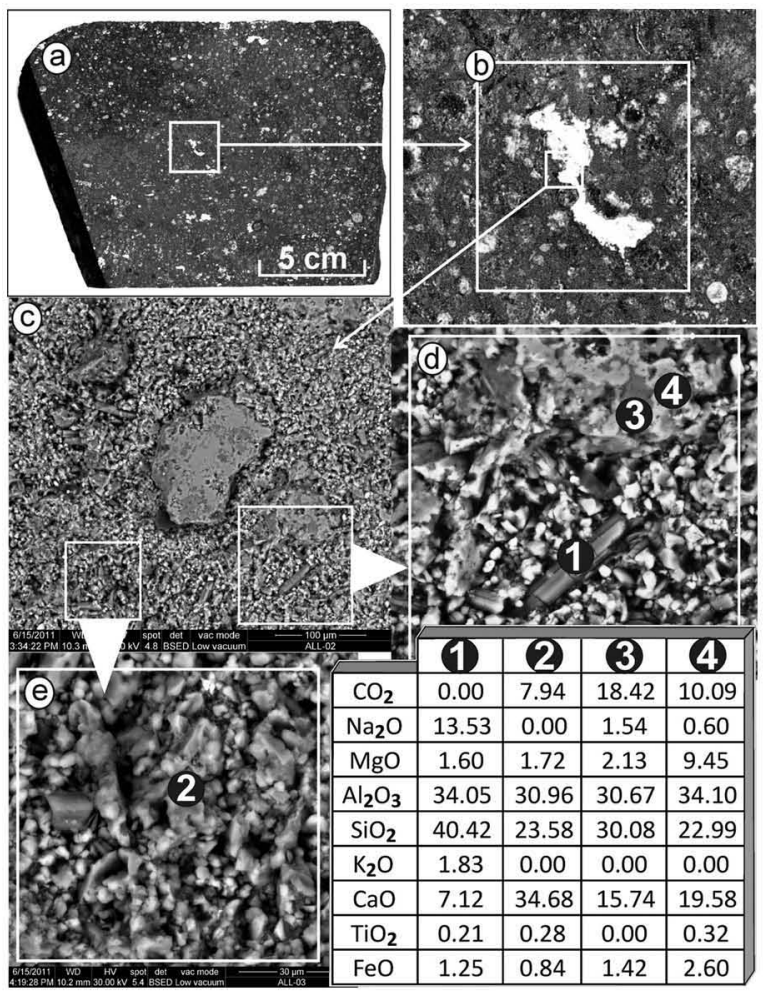

FIGURE 2 (a) Allende CV3 chondrite slice. (b) Detail of a white CAI inclusion. (c) BS-ESEM image with phenocrystals and matrix of Ca-Al-silicates. (d) \& (e) Details of the EDS spot analysis positions and table of EDS chemical analyses of these spots. masses as the best luminescent materials, that is, the Ca-Al-rich inclusions of white CAIS (Fig. 2b). Under the BS probe of the ESEM microscope, we observed the well-know large compositional and textural variability of the CAI phases (Fig. 2c).

Accordingly, we explored both phenocrystals (Fig. 2d-1) and background polycrystalline matrix (Fig. 2d-2,3) and (Fig. 2e-4). The EDS probe shows chemical compositions suggesting Ca-aluminosilicates such as anorthite and melilite and variable amounts of carbon, which mask the theoretical formulae (Fig. 2's table). Later, we use this initial exploration to perform spectral CL measurements by panchromatic plots and spot CL recordings.

\section{DISCUSSION}

We record spatially resolved and spectrally resolved CL measurements using spectral CCD detectors in high-sensitivity MonoCL3 photomultiplier with very high quantum efficiency, together with the intrinsic high luminescence sensitivity of the CAIS phases of Allende chondrite. The resultant spectral CL emissions overtake the 450,000 a.u. It is very interesting that just observing the large intensity of the CL emissions detected the large uniformity of the spectral CL curves along different CAI phases classified in situ by the BS and EDS analyses (Fig. 3a). Additionally, we experienced a bigger and last surprise in fitting these CL curves recorded from Allende CAI phases with other similar curves taken from terrestrial marble and limestone 


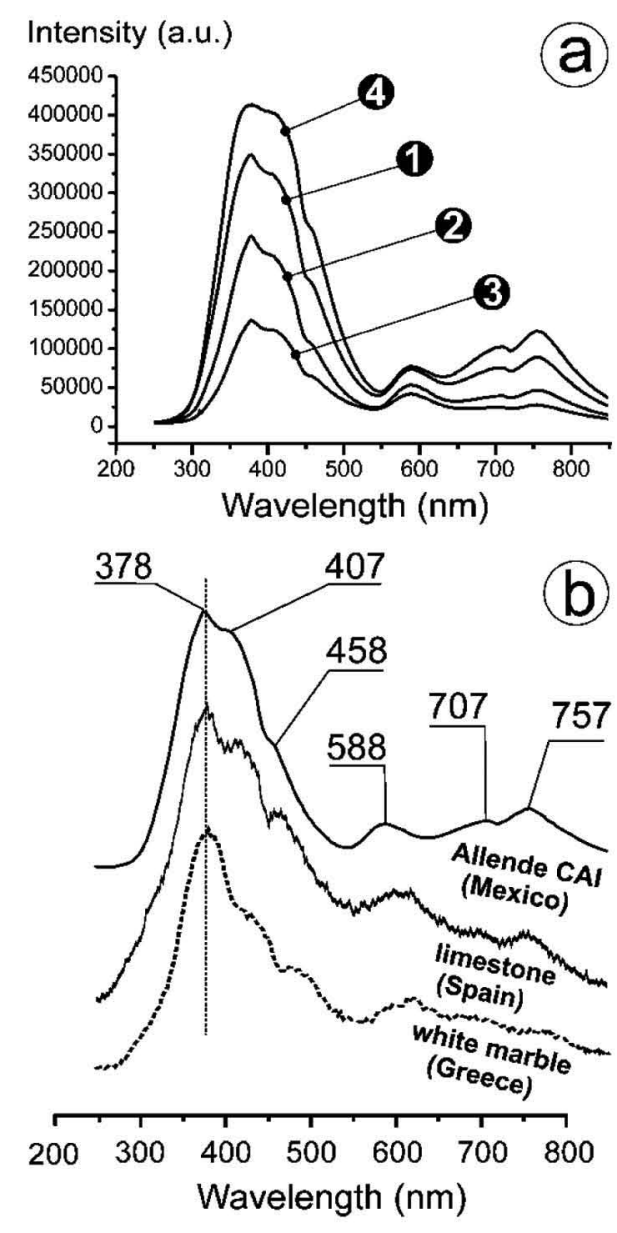

FIGURE 3 (a) Spectra CL plots taken from different CAl phases of the Allende $\mathrm{CV} 3$ chondrite. The experimental spectra CL curves are similar despite the different phases observed through the BSE grey tones and analyzed with the EDS probe. (b) CL-CAI Allende plot compared with terrestrial marble and limestone.

(Fig. 3b), that is, carbonated rocks formed by calcite microcrystals. A simplistic conclusion would be to state that we found calcite phase in the Allende CAIS, but we cannot detect calcite clearly by the EDS probe. These spectral CL curves of calcite-like shape need detailed explanations provided by our previous experimental knowledge from studying calcite luminescence together with some useful references. The common factor of these calcite-like CL curves, that is, in limestone and marble samples, is the existence of crystalline stress, which supports probable oxygen deficient centers (ODC) and nonbridging oxygen hole centers (NBOHC) in the calcite lattice. In sedimentary hydrated limestone or weathered marble, the hydroxyl groups (OH)- operate as oxygen-defect precursors, for example, $\mathrm{C}-\mathrm{O}$ or $\mathrm{Ca}-\mathrm{O}$ bonds, linked to grain surfaces producing large concentration of impurity defects at grain boundaries and cracks, very similar to the opals case. ${ }^{[17]}$ Two features support this theory, as follows. First, one feature is the general behavior of insulator materials without strong activators to enlarge their luminescence emissions of the UV-region of the spectrum under the analytical stress in cryogenic conditions (paper $340 \mathrm{~nm}$ ). Conversely, under stress, they restrain the emission intensities associated with point defects in the orange-red-IR region of the spectrum. Second, the burial supports the idea that the UV-band of the photoluminescence spectra of white marble surfaces being observed and that the soil acidity is a critical factor with a total absence of the blue-green emission $(380-530 \mathrm{~nm})$ at $\mathrm{pH}$ values lower than six. ${ }^{[15]}$ This feature supports our suggested model that since we removed the hydroxyl groups, as oxygen-defect precursors (more acidity) in the outers of the $\mathrm{CaCO}_{3}$ lattices, the stress and the UV-blue luminescence of the sedimentary hydrated limestone or weathered marble dropped. Concerning the spectra CL curves of the Allende CAI, we suggest a similar model assuming the following features: (i) more stress, that is, more CL intensity; (ii) less $(\mathrm{OH})^{-}$ groups, that is, endogenous geological origin without weathering; (iii) no clear detection of calcite crystals described in previous works as a secondary phase together with hydrous silicate glass; ${ }^{[1]}$ (iv) $\mathrm{C}-\mathrm{O}$ or $\mathrm{Ca}-\mathrm{O}$ bonds linked to the $\mathrm{Ca}-\mathrm{Al}$-Inclusion surfaces; (v) substitutional divalent cations, that is, $\mathrm{Mn}^{2+}, \mathrm{Fe}^{2+}$ in calcium crystallographic positions; (vi) existence of variable amounts of carbon element detected by EDS-ESEM (see EDS analyses in Figure 2's table); and (vii) existence of important amounts of calcium element. The model here enclosed predicts the existence of crystalline stress and unidentified crypto-clusters of calcite in the CAI phases. Additional survey by electron microscopy seems necessary to identify accurately the origin of the emission defects of these important spectral CL curves.

\section{CONCLUSIONS}

Calcium-aluminum-rich inclusions (CAIs) of Allende meteorite show intense spectra cathodoluminescence emission with a homogeneous curve pattern similar to other terrestrial CL patterns of stressed specimens of weathered marble and limestone in which hydroxyl groups could be involved. The inferred crypto-clusters of undetectable calcite 
associated to these CL emission centers will be the subject of further research.

\section{ACKNOWLEDGMENTS}

The authors are grateful to the Spanish project CGL2010-17108 for the financial support.

\section{REFERENCES}

1. Petaev, M. I.; Jacobsen, S. B. Petrologic study of SJ101, a new forsterite-bearing $\mathrm{CAl}$ from the Allende $\mathrm{CV} 3$ chondrite. Geochimica et Cosmochimica Acta 2009, 73(17), 5100-5114.

2. Grossman, L. Condensation in the primitive solar nebula. Geochimica et Cosmochimica Acta 1972, 36, 597-619.

3. Sears, D. W.; Mills, A. A. Thermoluminescence studies of the Allende meteorite. Earth and Planetary Science Letters 1974, 22, 391-396.

4. Barber, D. J.; Martin, P. M. The microstructure of minerals in coarse-grained Ca-Al-rich inclusions from the Allende meteorite. Geochimica et Cosmochimica Acta 1984, 48, 769-783.

5. Elgoresy, A.; Armstrong, J. T.; Wasserburg, G. J. Anatomy of an Allende coarse-grained inclusion. Geochimica et Cosmochimica Acta $1985,49,2433-2444$.

6. Stolper, E.; Paque, J. M. Crystallization sequences of Ca-Al-rich inclusions from Allende: The effects of cooling rate and maximum temperature. Geochimica et Cosmochimica Acta 1986, 50, 1785-1806.

7. Guimon, R. K.; Sears, D. W. G. Thermoluminescence and metamorphism of Allende and its CAl. Meteoritics 1986, 21, 381-382.
8. Steele, I. M. Digitized cathodoluminescence imaging of minerals. Scanning Microscopy 1991, 5(3), 611-618.

9. Fagan, T. J.; Kitou, H.; Tasai, Y. Cathodoluminescence of anorthite in two type b CAIS from Allende: Contrasting alteration patterns. L. P. I. Proceedings of the 71st Annual Meteoritical Society Meeting 2008, $5222,1$.

10. Fagan, T. J.; Guan, Y.; Macpherson, G. J. Al-Mg isotopic evidence for episodic alteration of $\mathrm{Ca}$-Al-rich inclusions from Allende. Meteoritics \& Planetary Science 2007, 42(7-8), 1221-1240.

11. Paque, J. M.; Beckett, J. R.; Ishii, H. A.; Aleon-Toppani, A. The formation of boundary clinopyroxenes and associated glass veins in type B1 CAls. Meteoritics \& Planetary Science 2009, 44(5), 665-687.

12. Calderon, T.; Townsend, P. D.; Beneitez, P.; Garcia-Guinea, J.; Millan, A.; Rendell, H. M.; Tookey, A.; Urbina, M.; Wood, R. A. Crystal field effects on the thermoluminescence of manganese in carbonate lattices. Radiation Measurements 1996, 26, 719-731.

13. Gaft, M.; Nagli, L.; Panczer, G.; Waychunas, G.; Porat, N. The nature of unusual luminescence in natural calcite $\mathrm{CaCO}_{3}$. American Mineralogist $2008,93,158-167$.

14. Galloway, R. B. Limestone: Some observations on luminescence in the region of $360 \mathrm{~nm}$. Radiation Measurements 2003, 37(2), 177-185.

15. Polikreti, K.; Christofides, C. The role of humic substances in the formation of marble patinas under soil burial conditions. Physics and Chemistry of Minerals 2009, 36(5), 271-279.

16. Garcia-Guinea, J.; Correcher, V.; Sanchez-Munoz, L.; Finch, A. A.; Hole, D. E.; Townsend, P. D. Luminescence emission band at $340 \mathrm{~nm}$ of stressed tectosilicate lattices. Nuclear instruments \& Methods Physics Research A 2007, 580, 648-651.

17. Stevens-Kalceff, M. A.; Thorogood, G. J.; Short, K. T. Charge trapping and defect segregation in quartz. Journal of Applied Physics 1999, 86(1), 205. 